\title{
Effect and mechanism of Src tyrosine kinase inhibitor sunitinib on the drug-resistance reversal of human A549/DDP cisplatin-resistant lung cancer cell line
}

\author{
KE ZHANG，XIAN WANG and HONGYAN WANG \\ Department of Thoracic Surgery, The First Affiliated Hospital, \\ Henan University of Science and Technology, Luoyang, Henan 471003, P.R. China \\ Received September 16, 2013; Accepted May 9, 2014
}

DOI: $10.3892 / \mathrm{mmr} .2014 .2440$

\begin{abstract}
The development of tumor cell drug resistance is the primary reason for treatment failure in lung cancer chemotherapy. Therefore, overcoming multidrug resistance is currently an urgent issue to be addressed in lung cancer treatment. Sunitinib is a tyrosine kinase inhibitor with confirmed inhibitory effects on tumor growth and metastasis; however, the effects of sunitinib and mechanisms of action in lung cancer multidrug resistance are yet to be determined. The present study was designed to examine the effects of sunitinib and the mechanisms underlying lung cancer multidrug resistance. It was observed that sunitinib was able to improve the sensitivity of A549/DDP lung cancer cells to cisplatin, enhance tumor apoptosis, arrest the cell cycle in G0/G1 phase, upregulate intracellular Rh-123 content, downregulate the expression of P-glycoprotein, multidrug resistance protein 1, multidrug resistance-associated protein 1, lung resistance protein, glutathione-S-transferase, ERCC1, survivin and Bcl-2 in tumor cells, phosphorylation of AKT and extracellular signal-regulated kinase (ERK), glutathione activity, and transcriptional activity of nuclear factor- $\mathrm{\kappa B}$, Twist, Snail and AP-1. The results demonstrated that sunitinib was able to reverse the multidrug resistance of A549/DDP lung cancer cells, which was possibly associated with the downregulation of multidrug resistance-associated gene expression and the inhibition of AKT and ERK phosphorylation.
\end{abstract}

\section{Introduction}

Lung cancer is the leading cause of cancer-associated mortality and was responsible for $\sim 1.38$ million deaths worldwide

Correspondence to: Professor Hongyan Wang, Department of Thoracic Surgery, The First Affiliated Hospital, Henan University of Science and Technology, 24 Jinghua Road, Luoyang, Henan 471003, P.R. China

E-mail: hongyan_wangly@126.com

Key words: multidrug resistance, lung cancer, tyrosine kinase inhibitor in 2008 (1). Lung cancer is divided by histological classification into non-small cell lung cancer and small cell lung cancer, the former of which accounts for $80 \%$ of lung cancer cases. Despite the poor prognosis of lung cancer, chemotherapy remains the primary strategy currently available for the treatment of advanced stage disease. Platinum drugs, including cisplatin and carboplatin, are the most frequently used chemotherapeutic drugs for these diseases although tumor cell resistance to platinum drugs is the main cause of clinical treatment failure. Therefore, overcoming drug resistance is urgently required to improve the clinical treatment efficacy for lung cancer patients (2).

Platinum drugs bind to the nucleotide bases of DNA to interfere with DNA synthesis, inhibit tumor cell proliferation and induce apoptosis. The development of drug resistance in tumor cells is a complex process. The mechanisms of tumor cell resistance to platinum drugs include the following two aspects: (i) Overexpression of multidrug resistance genes to reduce intracellular accumulation of the drug; and (ii) the improved anti-apoptotic ability of tumor cells. The in-depth investigation of the signaling pathways involved in these two mechanisms and the search for methods to overcome drug resistance are a primary focus of cancer research. It has been established that elevated Src kinase activity induces the overexpression of drug resistance-associated genes, and the abnormally hyperactive AKT and extracellular signal-regulated kinase (ERK) signaling pathways cause metastasis and drug resistance in lung cancer (3-6).

Src is not only a tyrosine kinase with a molecular weight of $60 \mathrm{kDa}$ and a member of the membrane-associated Src family kinases in cell protein tyrosine kinases, but also an important role in regulating cell proliferation, migration, signal transduction and other associated functions. Numerous studies have demonstrated the abnormal activation of Src tyrosine kinase in a variety of tumor tissues and cells, which has been confirmed to be closely correlated with tumor growth, metastasis and angiogenesis, and therefore provides a potential target for antitumor drugs. Previously, sunitinib, a Src tyrosine kinase inhibitor, has attracted notable attention for its close correlation with tumor multidrug resistance and ability to reverse the multidrug resistance of tumor cells through inhibition of Src tyrosine kinase activity (7-9). However, the effects of sunitinib 
and mechanisms of action in lung cancer multidrug resistance are yet to be determined and require further study.

\section{Materials and methods}

Cells and cell culture. A549/DDP cisplatin-resistant human lung cancer cells (Academy of Military Medical Science, Beijing, China) were cultured in RPMI-1640 media (containing $10 \%$ fetal bovine serum) in an incubator under $5 \% \mathrm{CO}_{2}, 37^{\circ} \mathrm{C}$ and saturated humidity conditions, followed by digestion with $0.25 \%$ trypsin-EDTA for sub-culturing. All of the experiments employed exponentially growing cells.

Determination of the effect of sunitinib on A549/DDP proliferation using the CellTiter-Glo luminescent assay. The exponentially growing A549/DDP cells $\left(5 \times 10^{4}\right.$ cells $\left./ \mathrm{ml}\right)$ were seeded into 96-well microplates, $100 \mu \mathrm{l} /$ well and cultured under $37^{\circ} \mathrm{C}, 5 \% \mathrm{CO}_{2}$ and saturated humidity conditions overnight to allow for cell adhesion. Sunitinib $(0,1,2,5,10,20$, 50 and $100 \mu \mathrm{M}$ ) was added to the corresponding wells and cultured for a further $24 \mathrm{~h}$. Next, following the CellTiter-Glo kit (Promega Corporation, Madison, WI, USA) instructions to detect cell viability the cells were lysed and transferred to black 96-well plates. The ATP chemical reaction induction reagent was added and mixed thoroughly and the luminescence was determined with a microplate reader (BioTek, Winnoski, VT, USA) after $10 \mathrm{~min}$.

Determination of the cytotoxicity of sunitinib on A549/DDP cells using the CellTiter-Glo luminescent assay. The exponentially growing A549 or A549/DDP cells $\left(5 \times 10^{4}\right.$ cells $\left./ \mathrm{ml}\right)$ were seeded into 96-well microplates, $100 \mu \mathrm{l} /$ well and cultured under $37^{\circ} \mathrm{C}, 5 \% \mathrm{CO}_{2}$ and saturated humidity conditions overnight to allow for cell adhesion. Cisplatin $(0,1,2,5,10,20$, 50 and $100 \mu \mathrm{M}$ ) was added to the corresponding wells. Based on this, 0,1 or $2 \mu \mathrm{M}$ sunitinib was additionally added and the culture was continued for $72 \mathrm{~h}$. The CellTiter-Glo kit instructions to detect cell viability were followed as described above.

Determination of Src phosphorylation level by western blot analysis. Sunitinib $(0,1$ or $2 \mu \mathrm{M})$ was added to the exponentially growing cells and cultured for $24 \mathrm{~h}$. The cell lysate was collected to extract the proteins and determine the protein contents using the bicinchoninic acid (BCA) method. An equal quantity of protein was obtained and separated in $12 \%$ sodium dodecyl sulfate-polyacrylamide gel (SDS-PAGE). The protein was transferred onto a polyvinylidene difluoride (PVDF; Millipore, Billerica, MA, USA) membrane and incubated with rabbit anti-human phosphorylated Src antibody (1:300; Biosource International, Inc., Camarillo, CA, USA) at $4^{\circ} \mathrm{C}$ overnight and then incubated with specific horseradish peroxidase (HRP)-streptavidin conjugated secondary antibody (Univ-bio, Shanghai, China) for $1 \mathrm{~h}$. The immunoreactive bands were then washed and developed using $\beta$-actin as the internal control (1:5,000; Sigma, St. Louis, MO, USA).

Determination of the effect of sunitinib on A549/DDP cell apoptosis using flow cytometry. Sunitinib $(0,1$ or $2 \mu \mathrm{M})$ was added to the exponentially growing A549/DDP cells and cultured for $24 \mathrm{~h}$. A total of $10 \mu \mathrm{M}$ cisplatin was then also added and the culture was continued for a further $24 \mathrm{~h}$. The cells were harvested and incubated with Annexin V-fluorescein isothiocyanate (FITC) and propidium iodide (PI) at room temperature away from light for $15 \mathrm{~min}$, and then the apoptosis rate was detected with a flow cytometer (Beckman Coulter, Brea, CA, USA).

Determination of the effect of sunitinib on cell cycle of A549/DDP cells by flow cytometry. Sunitinib $(0,1$ or $2 \mu \mathrm{M})$ was added to the exponentially growing A549/DDP cells and cultured for $24 \mathrm{~h}$. The cells were then harvested and incubated with PI (with RNase A) at room temperature away from light for $30 \mathrm{~min}$, and the cell cycle analysis was performed by a flow cytometer.

Determination of the effect of sunitinib on drug excretion and accumulation in A549/DDP cells by flow cytometry. Sunitinib $(0,1$ or $2 \mu \mathrm{M})$ was added to the exponentially growing A549/DDP cells and the cells were cultured for $24 \mathrm{~h}$. The cells were then harvested and incubated with $\mathrm{Rh}-123$ at room temperature away from light for $60 \mathrm{~min}$, or with phycoerythrin-P-glycoprotein (P-gp) at room temperature away from light for $30 \mathrm{~min}$. The intracellular Rh-123 content and cellular surface P-gp expression was then detected by a flow cytometer.

Determination of the effect of sunitinib on glutathione (GSH) activity in A549/DDP cells by biochemical assay. Sunitinib (0, 1 or $2 \mu \mathrm{M}$ ) was added to the exponentially growing A549/DDP cells and the cells were cultured for $24 \mathrm{~h}$. The cells were harvested and lysed, following the kit instructions to detect cellular GSH activity (Beyotime, Beijing, China).

Determination of the effect of sunitinib on multidrug resistance gene protein expression in A549/DDP cells by western blot analysis. Sunitinib $(0,1$ or $2 \mu \mathrm{M})$ was added to the exponentially growing A549/DDP cells and culture was continued for $24 \mathrm{~h}$. The cells were harvested and lysed to extract the proteins. The protein contents in the cell lysate were then determined using the BCA method. An equal quantity of proteins were obtained and separated in $12 \%$ SDS-PAGE, and then transferred onto a PVDF membrane and incubated with the respective monoclonal antibodies [anti-multidrug resistance protein 1 (MDR1), 1:800; anti-multidrug resistance-associated protein 1 (MRP1), 1:800; anti-lung resistance protein (LRP), 1:800; anti-glutathione-S-transferase (GST), 1:800; anti-ERCC1, 1:800; anti-survivin, 1:800; anti-Bcl-2, 1:800; anti-p-AKT, 1:300; and anti-p-ERK, 1:300] at $4^{\circ} \mathrm{C}$ overnight. Then, the cells were incubated with specific HRP-conjugated secondary antibody for $1 \mathrm{~h}$ and the immunoreactive bands were washed and developed using $\beta$-actin as the internal control (1:2,000; Sigma).

Determination of the effect of sunitinib on multidrug resistance-associated gene mRNA expression in A549/DDP cells by quantitative polymerase chain reaction (PCR) assay. Sunitinib $(0,1$ or $2 \mu \mathrm{M})$ was added to the exponentially growing A549/DDP cells and cultured for 24. The cells were then harvested and the total RNA was extracted using the TRIzol method, obtaining the cDNA by reverse transcription using a real-time PCR kit (Takara Bio, Inc., Dalian China). 
The primers used were as follows: sense: 5'-AGGAAGACA TGACCAGGTATGC-3' and antisense: 5'-CCAACATCG TGCACATCAAAC-3' for MDR1; sense: 5'-CTTCTGGAG GAATTGGTTGTATAGAAG-3', and antisense: 5'-GGTAGA CCCAGACAAGGATGTTAGA-3' for MRP1; sense: 5'-CAG CTGGCCATCGAGATCA-3' and antisense: 5'-TCCAGT CTCTGAGCCTCATGC-3' for LRP; sense: 5'-TTCCTTACT GGTCCTCACATCTC-3' and antisense: 5'-TCACCGGAT CATGGCCAGCA-3' for GST- $\pi$; sense: 5'-CCCTGGGAA TTTGGCGACGTAA-3' and antisense: 5'-CTCCAGGTA CCGCCCAGCTTCC-3' for ERCC1; sense: 5'-GAATTC ATGGGTGCCCCGACGTTGCC-3' and antisense: 5'-AGA TCTTTCTTCTTATTGTTGGTTTCC-3' for survivin; sense: 5'-TTGGCCCCCGTTGCTT-3' and antisense: 5'-CGG TTGTCGTACCCCGTTCTC-3' for Bcl-2; and sense: 5'-ATGGAAATCCCATCACCATCTT-3' and antisense: 5'-CGCCCCACTTGATTTGG-3' for glyceraldehyde 3-phosphate dehydrogenase. Following denaturation at $94^{\circ} \mathrm{C}$ for $3 \mathrm{~min}$, the primers were amplified under the following conditions for 40 cycles: $95^{\circ} \mathrm{C}$ for $5 \mathrm{sec}, 65^{\circ} \mathrm{C}$ for $35 \mathrm{sec}, 72^{\circ} \mathrm{C}$ for $60 \mathrm{sec}$, and extension at $72^{\circ} \mathrm{C}$ for $5 \mathrm{~min}$ after these cycles.

Determination of the effect of sunitinib on the transcriptional activity of transcription factors in A549/DDP cells using the dual luciferase reporter gene assay. A total of $3 \times 10^{4}$ cells were seeded in 24-well plates and cultured for $24 \mathrm{~h}$ to allow cell adhesion. Following the method as described in the manufacturer's instructions; nuclear factor (NF) $-\kappa B$, Twist, Snail and AP-1 luciferase reporter plasmids (Biominda, Tianjin, China) were added to each well to transfect the cells and then the cells were cultured for a further $6 \mathrm{~h}$ prior to washing off the plasmids that were not transfected into the cells. The media was then changed and 0,1 or $2 \mu \mathrm{M}$ sunitinib was added to continue the culture in an incubator for $24 \mathrm{~h}$. Finally, the activity of the luciferases was determined using a Dual-Glo ${ }^{\mathrm{TM}}$ Luciferase assay system (Promega Corporation).

Statistical methods. The experimental data are represented as the mean \pm standard deviation and analyzed with SPSS 11.5 software (SPSS, Inc., Chicago, IL, USA). Univariate analysis of variance was used for comparison and $\mathrm{P}<0.05$ was considered to indicate a statistically significant difference.

\section{Results}

Effect of sunitinib on A549/DDP cell proliferation. The CellTiter-Glo experimental results demonstrated that the inhibitory effect of sunitinib on A549/DDP cell proliferation was dose-dependent, it was identified that $1 \mu \mathrm{M}$ sunitinib did not have significant toxicity (inhibition rate $<5 \%$ ) on A549/DDP cells and $2 \mu \mathrm{M}$ sunitinib had low toxicity (inhibition rate, $10-15 \%$; Fig. 1). Thus, the 1 and $2 \mu \mathrm{M}$ sunitinib doses were selected for use in this study.

Sunitinib increases the sensitivity of A549/DDP cells to cisplatin. The CellTiter-Glo experimental results demonstrated that 1 and $2 \mu \mathrm{M}$ sunitinib decreased the $\mathrm{IC}_{50}$ of cisplatin for inhibiting A549/DDP cells; the $\mathrm{IC}_{50}$ of cisplatin was 11.35 and $55.22 \mu \mathrm{M}$ for inhibiting A549 cells and A549/DDP cells, respectively. The $\mathrm{IC}_{50}$ of cisplatin was 38.53 and $21.72 \mu \mathrm{M}$ for

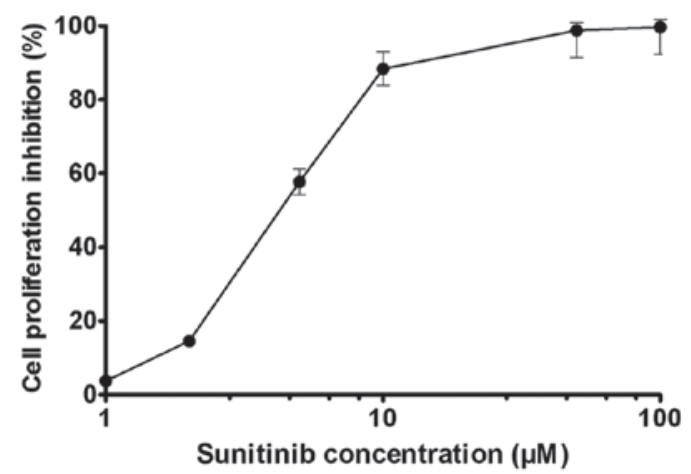

Figure 1. Effect of sunitinib on A549/DDP cell proliferation. The effect of sunitinib on A549/DDP cell proliferation was measured by CellTiter-Glo assay. The data are presented as the mean $\pm \mathrm{SD}$, the error bars indicate $\mathrm{SD}$, $\mathrm{n}=10 . \mathrm{SD}$, standard deviation

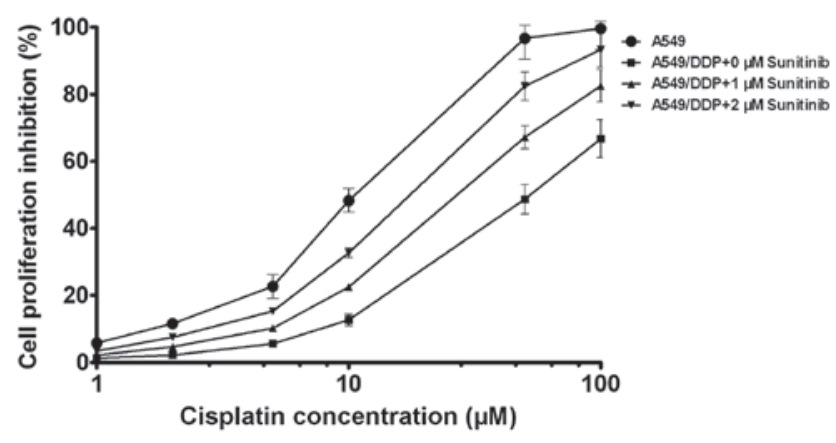

Figure 2. Effect of sunitinib on the sensitivity of A549/DDP cells to cisplatin. The effect of sunitinib on A549/DDP cell proliferation was measured by a CellTiter-Glo assay. The data was presented as the mean $\pm \mathrm{SD}$, the error bars indicate $\mathrm{SD}, \mathrm{n}=10$. SD, standard deviation.

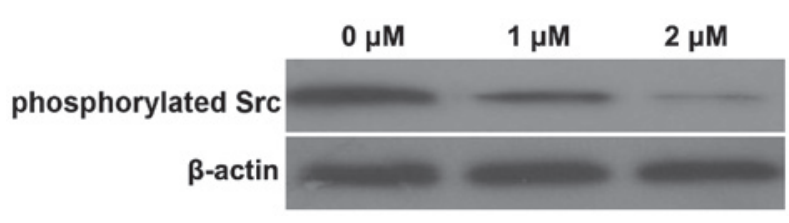

Figure 3. Effect of sunitinib on the Src tyrosine kinase activity in A549/DDP cells. The inhibitory effect of sunitinib on the phosphorylation level of Src tyrosine kinase in A549/DDP cells was measured by western blot analysis. $\beta$-actin served as the internal control.

inhibiting A549/DDP cells following treatment with 1 and $2 \mu \mathrm{M}$ sunitinib, respectively; and the reversal fold (RF) was 1.43 and 2.54, respectively (Fig.2).

Inhibitory effect of sunitinib on Src tyrosine kinase activity in A549/DDP cells. Western blot analysis demonstrated that the Src tyrosine kinase inhibitor, sunitinib, reduced the phosphorylation level of Src proteins in A549/DDP cells in a dose-dependent manner, therefore demonstrating that sunitinib was able to inhibit Src tyrosine kinase activity in tumor cells (Fig. 3).

Sunitinib inhibits P-gp expression in A549/DDP cells, increases cellular Rh-123 content, enhances apoptosis and 
A
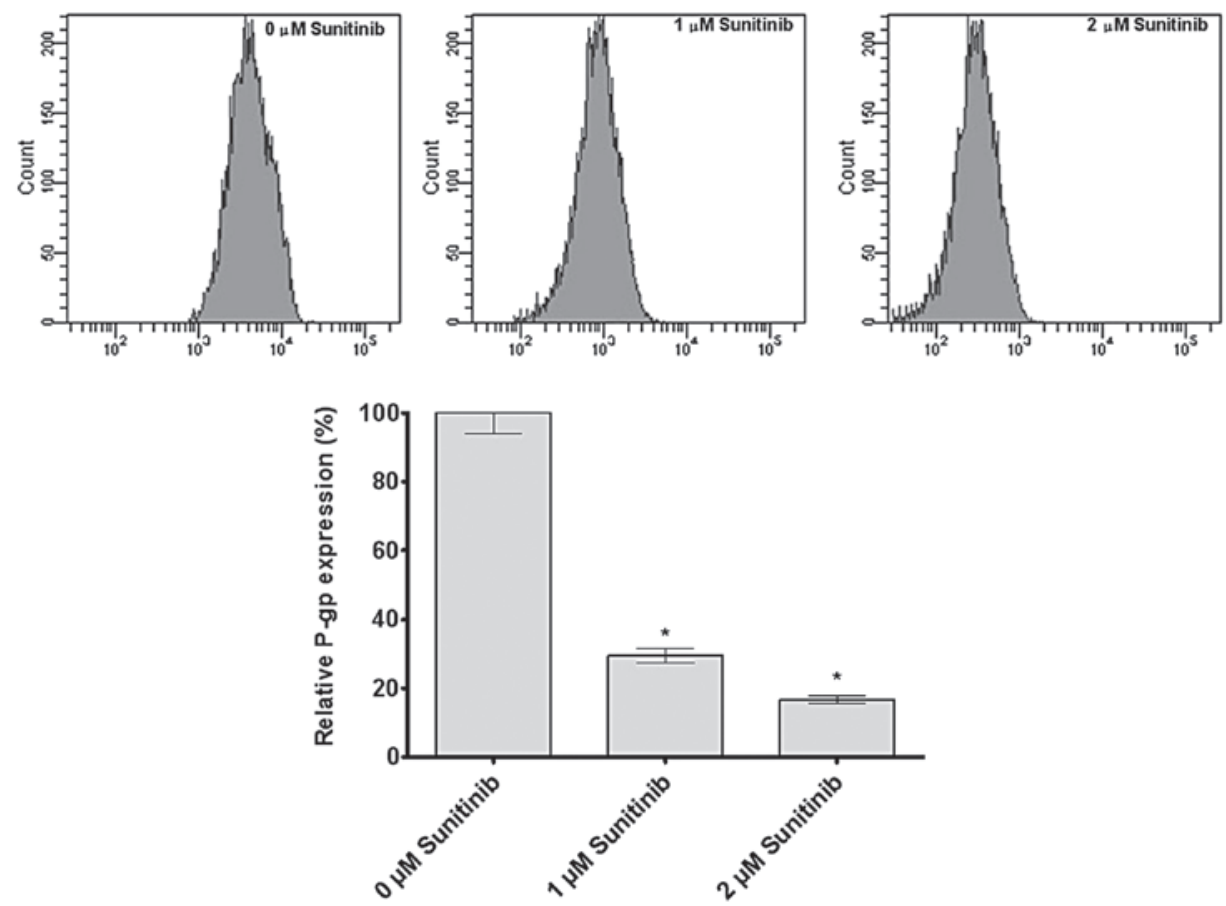

B
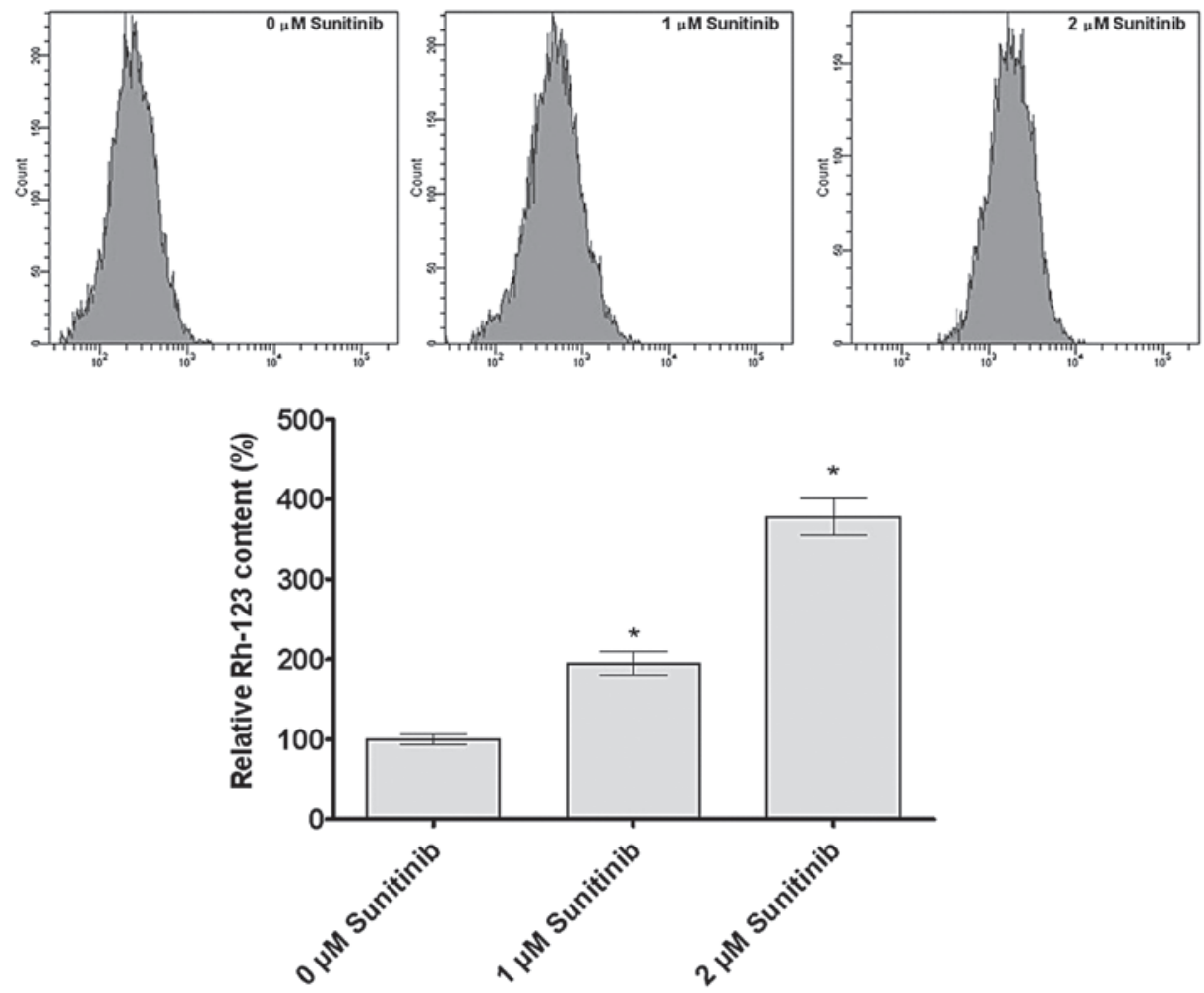

Figure 4. Effect of sunitinib on P-gp expression, cellular Rh-123 content, apoptosis and cell cycle of A549/DDP cells. The effect of sunitinib on (A) P-gp expression and (B) cellular Rh-123 content was measured by a flow cytometry assay. The data was presented as the mean \pm SD, the error bars indicate SD, $\mathrm{n}=3$. ${ }^{*} \mathrm{P}<0.05$, compared with the $0 \mu \mathrm{M}$ sunitinib group. $\mathrm{SD}$, standard deviation; P-gp, P-glycoprotein.

arrests cell cycle. As demonstrated by the flow cytometry results, sunitinib treatment resulted in a significant reduction in P-gp expression in the A549/DDP cells. When compared with the control group, the P-gp expression level was 29.5 and $16.6 \%$ in the 1 and $2 \mu \mathrm{M}$ groups, respectively. The sunitinib treatment also resulted in a significant elevation of Rh-123 content in A549/DDP cells, when compared with the control group, the mean fluorescence intensity of Rh-123 in tumor cells increased by 1.95 - and 3.78 -fold following treatment with 1 and $2 \mu \mathrm{M}$ of sunitinib, respectively (Fig. 4A and B).

The flow cytometry results also demonstrated a significantly higher apoptosis rate of A549/DDP cells following sunitinib treatment. The apoptosis rate in the 1 and $2 \mu \mathrm{M}$ group was 3.82- and 2.40-fold that of the $0 \mu \mathrm{M}$ group, respectively. 
C
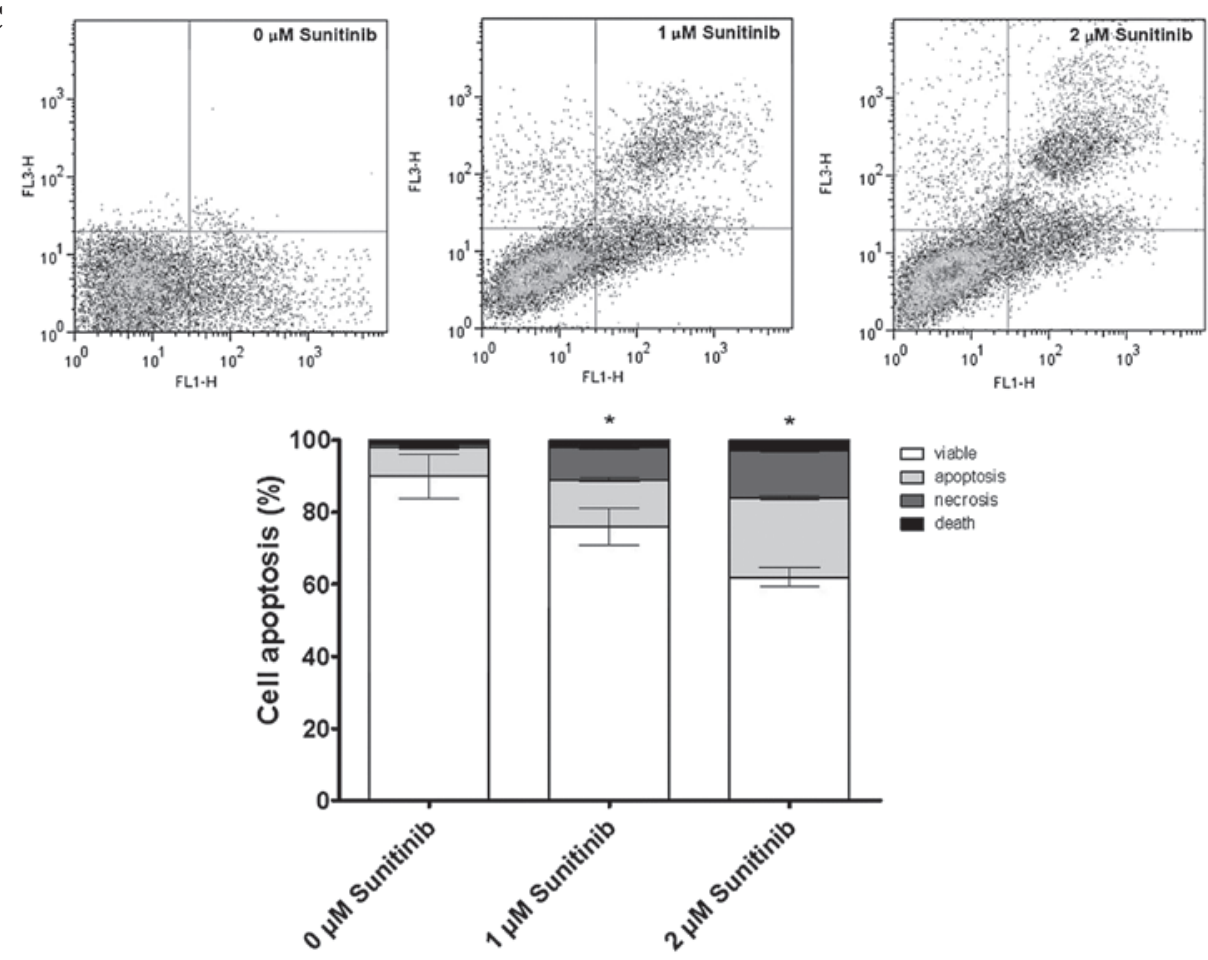

D
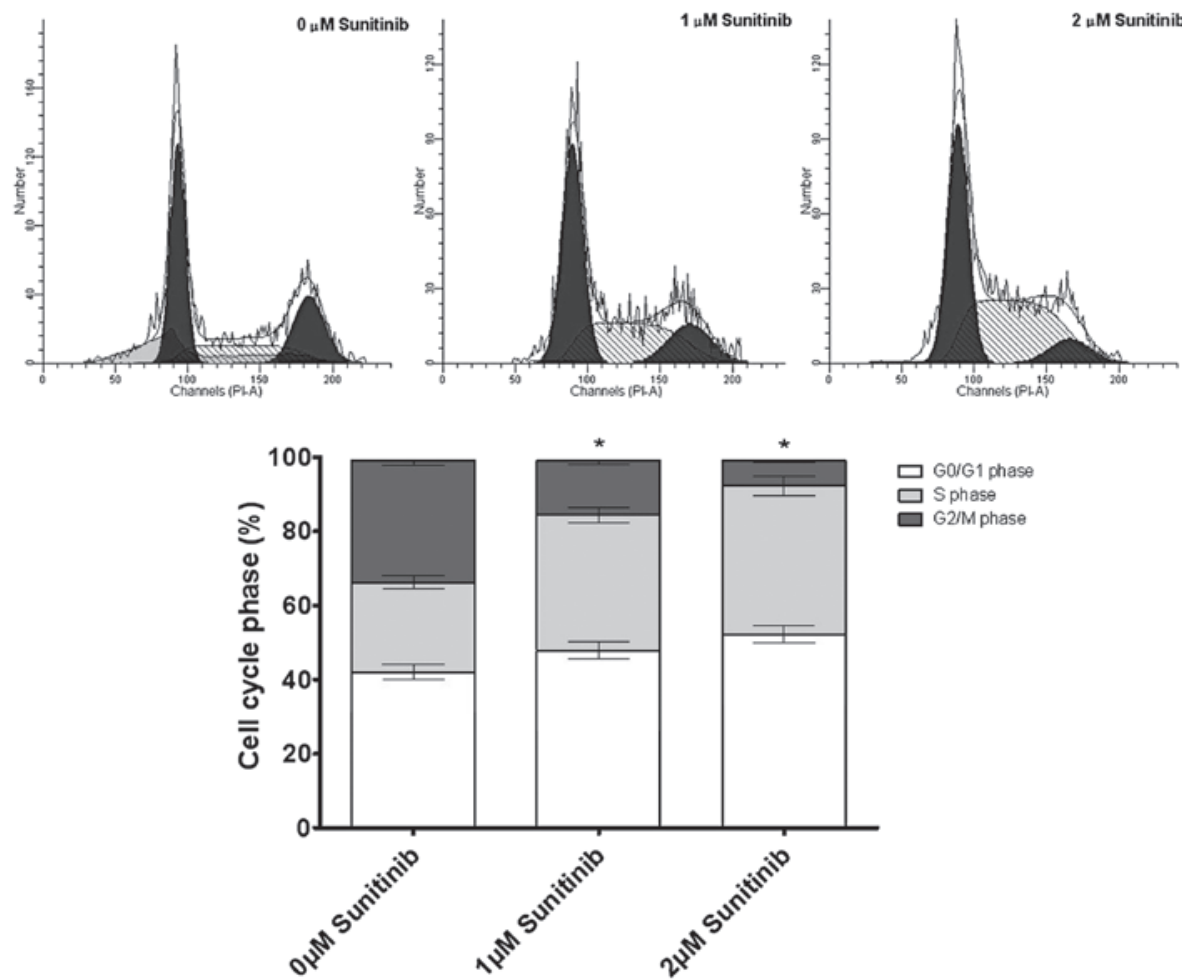

Figure 4. Continued. Effect of sunitinib on (C) cell apoptosis and (D) the cell cycle of A549/DDP cells was measured by a flow cytometry assay. The data was presented as the mean $\pm \mathrm{SD}$, the error bars indicate $\mathrm{SD}, \mathrm{n}=3 .{ }^{*} \mathrm{P}<0.05$, compared with the $0 \mu \mathrm{M}$ sunitinib group. SD, standard deviation.

Sunitinib treatment resulted in the arrest of A549/DDP cell cycle in $\mathrm{G} 0 / \mathrm{G} 1$ phase; the ratio of tumor cells in G0/G1 phase was 47.86 and $52.19 \%$ in the 1 and $2 \mu \mathrm{M}$ groups, respectively, which was significantly higher than the $41.97 \%$ in $0 \mu \mathrm{M}$ group (Fig. 4C and D), further demonstrating the ability of sunitinib to enhance apoptosis and arrest the cell cycle of A549/DDP cells. The results demonstrate that sunitinib was able to reduce drug excretion from tumor cells, increase drug content in tumor cells and arrest the cell cycle, resulting in the enhanced drug sensitivity and apoptosis of tumor cells.

Sunitinib downregulates the expression of multidrug resistance-associated genes. Western blot analysis demonstrated that sunitinib was able to reduce MDR1, MRP1, LRP, GST, 

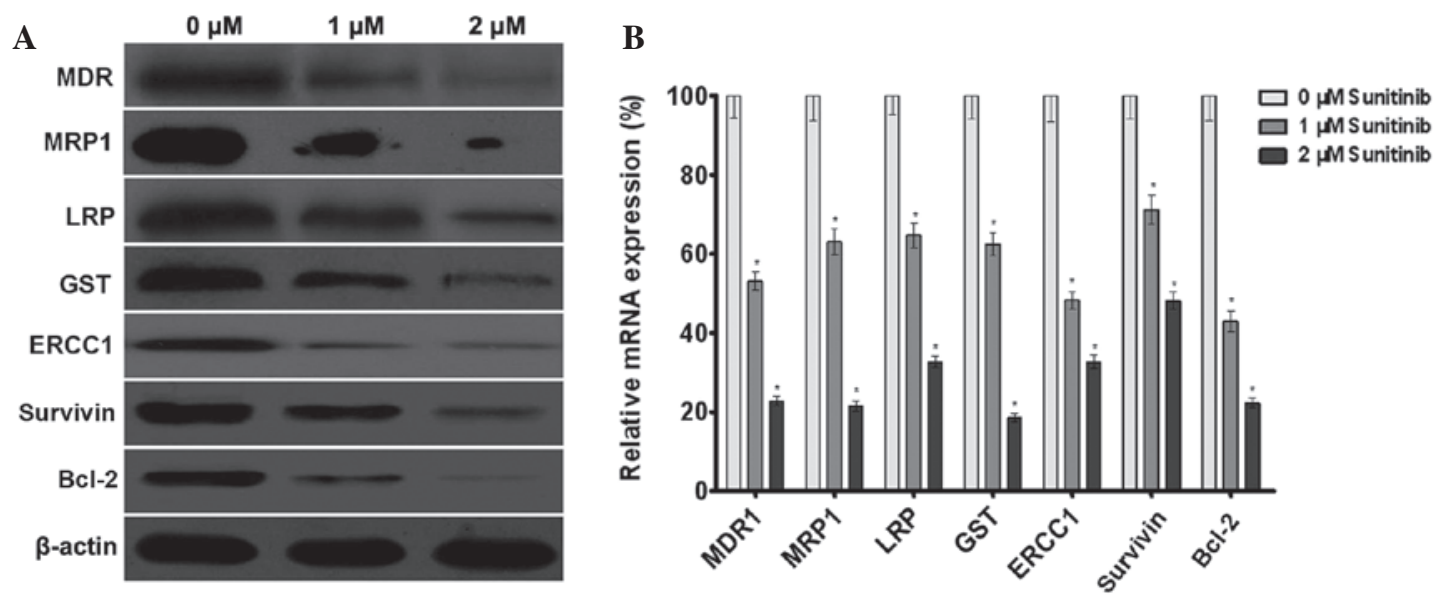

Figure 5. Effect of sunitinib on the expression of the multidrug resistance-associated genes in A549/DDP cells. (A) The effect of sunitinib on the protein expression of MDR1, MRP1, LRP, GST, ERCC1, survivin and Bcl-2 in A549/DDP cells was measured by western blot analysis. $\beta$-actin served as the internal control. (B) The effect of sunitinib on the mRNA expression of MDR1, MRP1, LRP, GST, ERCC1, survivin and Bcl-2 in A549/DDP cells was measured by quantitative polymerase chain reaction assay. Glyceraldehyde 3-phosphate dehydrogenase was used as the internal control. The data are presented as the mean $\pm \mathrm{SD}$, bars indicate $\mathrm{SD}, \mathrm{n}=5$; " $\mathrm{P}<0.05$, compared with the $0 \mu \mathrm{M}$ sunitinib group. MDR1, multidrug resistance protein 1 ; MRP1, multidrug resistance-associated protein 1; LRP, lung resistance protein; GST, glutathione-S-transferase; SD, standard deviation.

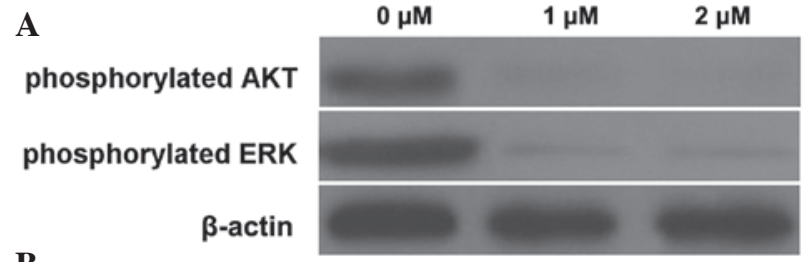

B

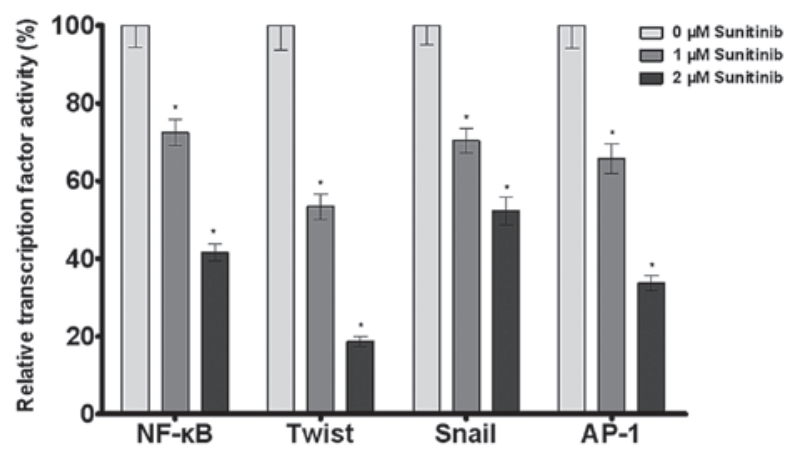

Figure 6. Effect of sunitinib on multidrug resistance-associated signal transduction pathways of A549/DDP cells. (A) The effect of sunitinib on the phosphorylation level of AKT and ERK in A549/DDP cells was measured by western blot analysis. $\beta$-actin served as the internal control. (B) The effect of sunitinib on the transcriptional activity of NF- $\mathrm{B}$, Twist, Snail and AP-1 in A549/DDP cells was measured by the dual luciferase reporter gene assay. The data are presented as the mean $\pm \mathrm{SD}$, bars indicate $\mathrm{SD}, \mathrm{n}=5 ;{ }^{*} \mathrm{P}<0.05$, compared with the $0 \mu \mathrm{M}$ sunitinib group. NF- $\kappa \mathrm{B}$, nuclear factor- $\kappa \mathrm{B}$; SD, standard deviation.

ERCC1, survivin and Bcl-2 protein level in A549/DDP cells in a dose-dependent manner (Fig. 5A), the results revealed that sunitinib downregulated multidrug resistance-associated protein expression in tumor cells, thereby enhancing the drug sensitivity of tumor cells. The qPCR results revealed that sunitinib downregulated MDR1, MRP1, LRP, GST, ERCC1, survivin and Bcl-2 mRNA levels in the A549/DDP cells following treatment with 1 and $2 \mu \mathrm{M}$ of sunitinib (Fig. 5B). The results demonstrated that sunitinib was able to downregulate
MDR1, MRP1, LRP, GST, ERCC1, survivin and Bcl-2 gene transcription in tumor cells, thereby enhancing the drug sensitivity of tumor cells.

Effect of sunitinib on multidrug resistance-associated signal transduction pathways in tumor cells. Western blot analysis revealed that sunitinib was able to reduce the phosphorylation levels of AKT and ERK in A549/DDP cells. The dual luciferase reporter gene assay results demonstrated that sunitinib downregulated the transcriptional activity of the transcription factors NF- $\kappa B$, Twist, Snail and AP-1. The results demonstrated that sunitinib regulated the transcriptional activity of NF- $\kappa B$, Twist, Snail and AP-1 by inhibiting AKT and ERK phosphorylation, leading to the inhibition of multidrug resistance gene expression and the reversal of tumor cell drug resistance (Fig. 6).

\section{Discussion}

The development of tumor cell multidrug resistance is the main cause of treatment failure of chemotherapeutic drugs; thus, investigating the mechanisms of tumor multidrug resistance and investigating potential solutions have important significance in improving the clinical efficacy of chemotherapeutic drugs. Studies have demonstrated that tumor cells may develop drug resistance through the following mechanisms: (i) Overexpression of ATP-binding cassette protein super family and other trans-membrane protein genes to pump the drugs out of tumor cells and reduce the intracellular drug concentration; (ii) overexpression of detoxification and DNA repair enzymes to reduce the efficacy of cytotoxic drugs; (iii) downregulation of the expression of drug target molecules to reduce drug sensitivity; and (iv) activation of tumor suppressor genes to inhibit drug-induced apoptosis. Among them, the most extensively studied mechanism is drug resistance induced by the overexpression of multidrug resistance genes. The expression of these proteins reduces the drug concentration in tumor cells or in the vicinity of the drug 
targets, rendering it impossible for the administered drugs to reach the treatment sites effectively and leading to the subsequent development of drug resistance (10).

Multidrug resistance protein 1 (MDR1) as an energy-dependent transmembrane glycoprotein is extensively expressed in lung cancer tissues and expressed at moderate to low level or not expressed at all in normal lung tissues. MDR1 protein may function as a drug pump. When overexpressed in tumor cell membranes, MDR1 protein binds to the antitumor drug and pumps the intracellular drugs out of the cells actively using the energy released by ATP hydrolysis, leading to a reduction of intracellular drug concentration and drug resistance. MRP1 and LRP are important drug resistance genes that have been identified in recent years and act as major vault proteins (11). They were first identified in lung cancer and have since been demonstrated to be overexpressed in a variety of drug-resistant cell lines (12). MRP1 affects the intracellular transport and distribution of drugs, leading to drug resistance (13).

GST is able to eliminate cisplatin-induced DNA insertion. Simultaneously, the peroxidase activity of GST and GSH reduces cisplatin-induced peroxide and covalent conjugates. Therefore, GST, GSH and MDR1 exert synergistic effects; namely, MDR1 pumps the cisplatin-glutathione conjugates, catalyzed by GST and GSH, out of the cells and has an important role in cisplatin resistance. Nucleotide excision repair is an important mechanism responsible for cisplatin resistance. ERCC1 is a crucial pathway for DNA repair and has an important role in the repair of cisplatin-induced DNA damage; ERCC1 has been proven to be an important drug resistance gene in tumors. As survivin and Bcl-2 are two key genes in the regulation of apoptosis, that inhibit apoptosis synergistically, the downregulation of survivin and Bcl-2 expression has emerged as an important pathway for reversing tumor multidrug resistance (14-16).

The drug-resistant A549/DDP cells that are screened from the A549 human lung cancer cell line by continuous exposure to cisplatin are an excellent tool for investigating multidrug resistance. The importance of Src tyrosine kinase in tumor formation and development is well established, and it has been demonstrated that the overexpression of Src leads to the development of cancer. Furthermore, recent studies have demonstrated that Src kinase is also involved in tumor cell resistance to chemotherapeutic drugs, as the Src tyrosine kinase inhibitor increases tumor cell sensitivity to chemotherapeutic drugs and may even reverse drug resistance. Although the association between Src tyrosine kinase and tumor drug resistance remains unknown, the roles of Src tyrosine kinase inhibitors in different types of drug-resistant tumor cells are worthy of further study.

The present study demonstrated that the Src tyrosine kinase inhibitor sunitinib was able to inhibit Src kinase activity in A549/DDP cells, as evidenced by the reduced level of Src phosphorylation. The CellTiter-Glo assay results revealed that this inhibitory effect was further converted to enhance cell sensitivity to cisplatin. One of the main mechanisms responsible for A549/DDP cell drug resistance is the overexpression of MDR1 and MRP1, which leads to the potentiation of the cell's ability to excrete the drug and the consequent reduction of drug concentration in the cells. Therefore, the reversal of drug resistance may be associated with the restoration of drug accumulation in these cells. As Rh-123, a substrate of P-gp, indicates the expression of this protein in cells and the ability of cancer cells to pump out the drug $(17,18)$, the effects of sunitinib on Rh-123 content in the cells was investigated using flow cytometry. The results demonstrated that sunitinib treatment resulted in the significant elevation of $\mathrm{Rh}-123$ content in the cells, suggesting that the inhibition of Src tyrosine kinase activity and the reversal of drug resistance of A549/DDP cells are associated with the inhibition of drug excretion from the cells, the increased level of intracellular drug accumulation, and the consequent arrest of the cell cycle and enhancement of apoptosis, which is consistent with our hypothesis. Western blot analysis demonstrated that sunitinib treatment resulted in downregulation of the expression levels of ERCC1, survivin and $\mathrm{Bcl}-2$ protein in the cells; while the quantitative PCR results suggested that sunitinib-induced downregulation of the expression of the aforementioned proteins was likely achieved by downregulating the level of mRNA transcription.

Furthermore, it was observed, through studies on signal transduction pathways in tumor cells, that sunitinib was able to inhibit the phosphorylation of AKT and ERK, leading to the subsequent downregulation of the transcriptional activity of NF- $\mathrm{B}$, Twist, Snail and AP-1. It was therefore hypothesized that sunitinib is able to inhibit the expression of multidrug resistance genes and reverse tumor drug resistance by inhibiting the aforementioned signal transduction pathways. The present study demonstrated that sunitinib was able to reverse the A549/DDP cell multidrug resistance and that the mechanisms may be associated with the downregulation of multidrug resistance gene expression in these cells and the restoration of intracellular drug accumulation. The results of this study have provided the experimental basis for the application of drugs targeting Src tyrosine kinase and the clinical reversal of lung cancer multidrug resistance.

\section{References}

1. Dearing KR, Sangal A and Weiss GJ: Maintaining clarity: Review of maintenance therapy in non-small cell lung cancer.. World J Clin Oncol 5: 103-113, 2014.

2. Hill C: Cancer prevention and screening. Bull Cancer 100: 547-554, 2013 (In French).

3. Chen YT, Feng B and Chen LB: Update of research on drug resistance in small cell lung cancer chemotherapy. Asian Pac J Cancer Prev 13: 3577-3581, 2012.

4. Curry NL, Mino-Kenudson M, Oliver TG, et al: Pten-null tumors cohabiting the same lung display differential AKT activation and sensitivity to dietary restriction. Cancer Discov 3: 908-921, 2013.

5. Park YH, Kim SU, Lee BK, et al: Prx I suppresses K-ras-driven lung tumorigenesis by opposing redox-sensitive ERK/cyclin D1 pathway. Antioxid Redox Signal 19: 482-496, 2013.

6. Salminen A, Lehtonen M, Suuronen T, et al: Terpenoids: natural inhibitors of NF-kappaB signaling with anti-inflammatory and anticancer potential. Cell Mol Life Sci 65: 2979-2999, 2008.

7. Peterson-Roth E, Brdlik CM and Glazer PM: Src-induced cisplatin resistance mediated by cell-to-cell communication. Cancer Res 69: 3619-3624, 2009.

8. Reynolds C, Spira AI, Gluck L, et al: Sunitinib malate in previously untreated, nonsquamous, non-small cell lung cancer patients over the age of 70 years: results of a Phase II trial. Invest New Drugs 31: 1330-1338, 2013.

9. Wei L, Dai Q, Zhou Y, et al: Oroxylin A sensitizes non-small cell lung cancer cells to anoikis via glucose-deprivation-like mechanisms: c-Src and hexokinase II. Biochim Biophys Acta 1830: 3835-3845, 2013. 
10. Melguizo C, Prados J, Luque R, et al: Modulation of multidrug resistance gene expression in peripheral blood mononuclear cells of lung cancer patients and evaluation of their clinical significance. Cancer Chemother Pharmacol 71: 537-541, 2013.

11. Zhang B, Liu M, Tang HK, et al: The expression and significance of MRP1, LRP, TOPOII $\beta$, and BCL2 in tongue squamous cell carcinoma. J Oral Pathol Med 41: 141-148, 2012.

12. Li XQ, Li J, Shi SB, et al: Expression of MRP1, BCRP, LRP and ERCC1 as prognostic factors in non-small cell lung cancer patients receiving postoperative cisplatin-based chemotherapy. Int J Biol Markers 24: 230-237, 2009.

13. Wang J, Zhang J, Zhang L, et al: Expression of P-gp, MRP, LRP, GST- $\pi$ and TopoII $\alpha$ and intrinsic resistance in human lung cancer cell lines. Oncol Rep 26: 1081-1089, 2011.

14. Banerjee A, Qian P, Wu ZS, et al: Artemin stimulates radio- and chemo-resistance by promoting TWIST1-BCL-2-dependent cancer stem cell-like behavior in mammary carcinoma cells. J Biol Chem 287: 42502-42515, 2012.
15. Han Y, Wang XB, Xiao N and Liu ZD: mRNA expression and clinical significance of ERCC1, BRCA1, RRM1, TYMS and TUBB3 in postoperative patients with non-small cell lung cancer. Asian Pac J Cancer Prev 14: 2987-2990, 2013.

16. Liu JL, Wang Y, Jiang J, et al: Inhibition of survivin expression and mechanisms of reversing drug-resistance of human lung adenocarcinoma cells by siRNA. Chin Med J (Engl) 123: 2901-2907, 2010

17. Munić V, Kelnerić Z, Mikac L and Eraković Haber V: Differences in assessment of macrolide interaction with human MDR1 (ABCB1, P-gp) using rhodamine-123 efflux, ATPase activity and cellular accumulation assays. Eur J Pharm Sci 41: 86-95, 2010

18. Wei HB, Hu J, Shang LH, et al: A meta-analytic review of ERCC1/MDR1 polymorphism and chemosensitivity to platinum in patients with advanced non-small cell lung cancer. Chin Med J (Engl) 125: 2902-2907, 2012. 